\section{Zur Frage der Sozialisierung der chemischen Industrie.}

Von Profegsor Dr. H. Grossmans.

(Finger. 17.; 2 1019)

Die Sozialisierungsirage; über deren Bedeutung für die chemisohe Industrio Deutschlands und die Chemiker selbst orst unlängst von Dr, I phrai m in dieser Zeitachrift') beriohtet worden ist, stellt siah immer mehr als die wrichtigete Forderung dar, die von don Arbeitor. maseen gegenwïrtig erhoben wird, obwoll man kanm wird sagen können, daß diese zum Teil nioht übermäDig klar durchdachton und geotellten Forderungen von den großen Massen selbst angegangen aind. Vielmebr dürtte os sich in der Ifauptsache wohl unz Anspríabe handeln, die von einzelnen sozialistischen Theoretikern in den leteten Monaten formuliert worden sind, und die zweifellos auch erheblich mit daxu beigetragen haben, dio Arbeiterkreise nicht zar Ruho kommen zu lassen.

Jo weniger man sich aber über den Begriff und die Folgen der Sozialisierung in den einzelnen Gewerben kJar ist, am so stärker wirkt eben diesea neueste Schlagwort der Rovolution in einer so orregten Zeit, in der es einer kühl überlogenden Kritik fast obenso whwer gemacht wird, durohzudringen, als es etwa in den ersten Triegamonaten der Fall geweaen ist. Es ersoheint jedoch auch gegen. wätig, und jetzt vielleicht noch mehr als jemals zuvor, als unbedingt notwondig, vor den neuen Gefalurer. im Innern dio Augen nicht zu versohließen, die cine überstürzto Sozialisierung besonders auch für die chemiscbe Industrie im Gefolge haben wiirde.

Um diese Gefahren klar zu erkennen, ist es aber auch notwenditg. dab die deutsohen Chemiker, und zwer Uuternehmer wio Angestellte in gloicher Weise, die soxialistischen Gedankengänge solbständig und

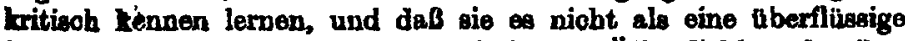
Zoitverschwendung betrachten, auch in der Offentliolukeit filir ihre ebrejohenden Ansichten einzutreten. Is mub daher jedenfalls mit deen geistigen Widerstando gegen solohe verhängnisvollen Theorien, donen konsequente Durchftilurung letzten Fondes auch die Arbeiter welbst am meisten schädigen wiurdo, einmal begonnen werden, und unter keinen Umstënden dart etwa eine Vogel Straußpolitik befolgt weardeo, die sicherlich nur clazu führen könnte, der ausländischen Bonkurrenz dio Wege noch mehr zu ebnen. DaB man im Auslande Lber diase Soxialigierungspläne, die auch vor der doutschen chemjwhen Industrie nioht Halt machen wollen, eine nur wenig verhüllte Bronde empfindet, ersoheint nach Berichten a.us versohiedenen Lladern jodeafalls unbedingt ioher.

Wenn man jedooh bedenkt, da B in der Tat auch das Wohl dor deutohen Arbeiter in der chemiechen Industrie anf dem wirtschattichen *edoiben der Untemehmungen beruht, so wird man aus diesem hronde erst reoht auf die verhängnisvollox Folgen jener Sozialiiangobestrebungen auf Grund theoretischer Erwägungen immer rioder zurückkommen mliesen. Auf dio großen Gefahren, die der bomischeu Industrio von einex überstirrten Soxialisierung drohen, abe ich erst unlëngst an anderer Stelle hingewiesen ${ }^{2}$. Deshalb seil - gestatset, in folgenden nur einige kritische Bemerkungen zu den Riohtlinien fir ein sozialistisohes Artionsprogramm" zu maohen, a dox zurzeit wohl sngesehenste Theoretiker des deutschen ouialismus, Karl Ka uts ky, als Beilage in dar Nummer des "Vor. inte" voin 2./2. d. J. veroffentilicht hat

Solbot wer dem soxialistisohen Gedankenkrois in wirtsobattlicher losicht stets ferngestanden hat, wird zugeben mitresen, dac in Hichtlinion manohe Bätzo enthalten sind, denes auch dio ver des Baxialismue wenden zuotimmon könen. Das gilt ganz wondars ran der noumsohränkten Aufforderung Ks utskys, - Produktion a heben. Fr bezetohnet diew Forderung mitc wht als dis dringandote Autgabe der Gegenwart und gleiohzeitig ha als die Vorbedingung oines jedion Voronohes einet Sonialisierung - Produltion. Die Durchfübrung der Soxielislerung aber

(1) Angew. Chem. 32, IX, 49 [1919].

1 Chem.2tg. 1919 Nr. 10. orscheint ihm goradeza als dio wiobtiggto Aufgabo des noven de utschen Stastos.

Welohe Aussichten eröffnen sioh nun aber in dieser neven tra der Industrie? Wenn man die Richtlinien aufmerkesm studiert, so findet man immer nur wioder die alten Forderungen der Vorstaricliohung, die dudurch wabrlioh nicht sobmackbatter ersoheinen, $\mathrm{da} B$ man sie mit der Bildung von $\mathrm{Z}$ wangse y ndi kate $\mathrm{n}$ verknupfon will. Denn Kautsky orklärt in nicht miBzuverstehendar Weise, daB jene Produktionszweige, deren sofortige Sozialiaierung nioht möglich ist, soweit sie dazu goeignet sind, durch don Staat zwanga. weise syndiziert werden sollen. Man darf allerdinge wobl annehmen, dab auch die großo Mebrzahl der gemäBigten Sozialister die chemischo Industrie vorläufig nooh nicht als ,sozialisierungsreif" aneehen wird, da B man aber diese Industrie jedenfalls mit staatlichen Zwangssyndikaten im Sinne Ka utsky zu beglüuken versucten wird. Wie wenig Freiheit jedoch in einom solohen Zwangowyndirat wird bestehen können, ergibt sich aus dén weiteren Forderungen, die im folgenden wörtlich wiodergegeben sind, um zu reigeu, ein wie schwerfalliger Organismus auch der chemisohen Industrio unter Umatänden zugemutet werden soll, eine Organisation, die zweifellas nicht dazu wird beitragen könuen, dio Arbeitsfreudigkuit und Unternehmungoluat wio das Bestreben, teohnische Neuerungen in Angriff zu nehmen und wie frtiher systemutisch an fördern, nach irgendeiner Richtung hin zu heben. "Das Syndikat hat dio Beschafing der Rohstoffe, den Absatz der Produkte, sowie die Rogelung der Produktionsbedingungen zu besorgen. Es hat suoh dus Becht, uberfluseigo oder unrationelle Botriebe stillzulegen. In soine Ioitung verdon gewählt zu einem Viortel Vertreter der Unternehmer, zu einen Viortol Vertreter der Arbeiterrate, endlich za einem dritten Viertel die der organisierton Kons u menten des Produltionszweiges") -. otwa Industrielle, wenn der Produktionszweig Produktionsmittel produziert, oder Vertreter von Konsumgenossenochaften und Gomeinden, wenn er Kousummittel berstellt. Das letzte Viertel wird gebildot von Vertretern des Staates, dio das Gesamtinteresso repräsentieren."

Ob ein solcher Debattierkl $u b$, in dom naturgem $3 B$ auch noch stets dio Gefo.hr besteht, daB wichtige Betriebsgehoimniesse verletzt werden kónues, gerade sehr förderlich erscheint, um den wirtschaftlichen und tochnischen Fortschritt in Deutschland zu begünstigen, nürfte wohl außerordenilich zweifelhaft erscheinen. Man vergegenwär. tige sich einmal prabtisch, zu welchen Folgen eine solche Ma Bnahme in einom modernen Großunternehmen, wit etwa der Badischen Anilinund Sodafabrik, der Höchstor Farhwerke usw. fuhren muB, wenn man jene zur eigentliohen Leitung ganzer Industrien berufenen Körpersohaften wählen wollte, ganx abgesehen davon, ob os sehr leicht wein wrürde, die geeigneten technisohen und wirtsobaftlichen Sachverständigen aus den rerschiedener Kreisen heranziehen zu können. Eu ersoheint das nämlich um so schwierigor, wenn man bedenkt, da $\beta$ auch für die einzelnen Unternehmungen von Kautgky nooh folgende Forderungen exhoben werden. „Innerhalb der einzelnen Bo triebe werden dem Unternehmer Arbeiterausachtissa oder Arbeiter rä te zur Seito gestellt, die die Durchführung der Beschliteno des Byadikats uberwachen und dahin wirken, da.B wio mogliohst sweckmüBig und unter Wahring der Arbeiterintereseen durobgefliht werden. Dds passive Wahlrecht zut den Betriebsarbeiterrilten haben auch Personen, die außerhalb des Betriebes stehen und vom Untornehmer Okonomisch unabhängig sind, wie etwr Arzte und Angeatellto von Arbeiterorganisetionen. Xhnliohe Arbeiterrito sollen auch in den niobt syndiziarten Betrieben eingerichtet worden."

Anoh diese Organisation, dio in ginotignten Fallo siob nux auf vielo Reden beschrikinen witrdo, düfto der groBen Mohrzahl dor ohemischen Unternehmer, und zwar mit vollem Recht, hoohst unaympathiseh ersobeinen. Jedenfalls darfte wioh der ganco Betriob

7) Wer weil, welche chomisthe Strofle trie Sohwofoledure, Salpoterniure, Sods usw. aufwaison, wird die Organisation der genannten Vertreter als eino beconders twaig gliboldiche Fonderung leicht exkennon. 
weosutiloh schwerfalliger gestalten, da ja stets notwondig sein wirde, durch sohwierige Kompronißverhandlungen zu einer Finheitliobkeit in den nun einmal reoht versohiedesien Ansohauungen der neven eigentlichen "Fabrikleiter" in bezug auf alle nouen MaBnahmen za golangon. Von einer ruhigen Entwioklung der Industrie, dio gerade jetzt besonders notwendig erschrint, dürfte jedenfalls bei der Durchführung soicher Pläne keine Rede nehr sein, und daher kann man auch wohl schon jetzt ohne Gefahr, eine Widerlegung befürchten zu müssen, die Prophezeiung wagen, daß bei Durchführung dieser Vorschläge der mit den vielen notwendig werdenden Wablen verbundene Geist der Unruhe und der Kabalen in cien einzelnen Werken wie in der ganzen Industrie in Permanenz erklärt werden würde. Das Interesse an ellbständigen Erfindungen aber würde in einem solchen System bestimmt nicht genïgend zi: Geltung koumen, und es dürtte daher bei weiterem Fortschreiten auf diesen geführ. lichen Bahnen die schon jotzt nicht geringe Gefahr ciner Abwanderung der deutachen Chemiker aus Deutschland um so brennender worden. Denn es werden sicherlich nicht die schlechtesten Köpfe win, die es ablehnen wärden, unter einem Zwang, wie ihn die Durch. fibrung des Kautsk y schen Programms herbeiführen muB, druernd waiter au arbriten. Einstmals hat Doutachland in der Realstionszeit nash den Freiheitskriegen und nach dem Mrillingen rior Rovolution von 1848 oine große Zah] von tilichtigen Kräften verloren, weil jene MännaY in einem untreien $\mathfrak{l}^{2}$ olizei- und Obrigkeitastatat nicht ferncr loben wollten, noch konnten. Es besteht aber auch jetzt in kaum viol geringerem Grade die Gefahr, daß wir in Deatschland eine Wiederholung dieser Verhältnisse erlebei, fofern eine einseitige Parteirichtung auch im Wirtschaftsleben zur Herruchaft gelungen wïrde, uie den berschtigten Freiheitsbedürfuisson dess nicht auf sozialistischem Boden stebenden Individuums trotz aller Freiheitsreden doch allsu viele und lästige Schranken aufzuerlegen tranhtet.

Ka u ssky erklärt allordings, daß die neuen Stastabetriche nicht nach der Schahlone der bisherigen Betriebe einer zentralisierten Bureaukratie eingerichtet werden sollen. Diese Staatsbetriebe wären vielmehr umzuformen und zu dezentralisieren und ihren Leiutungen möglichsto Selbständigkeit zu gewähren. Nach Möglichkeit soliten ferner richt einzelne Betriebe, sondorn gunze Industriezweige verstuatlicht werden. Hierbei sollte ebenfalls wioderın eine kollegiale Verwaltung oingerichtet werden, indem die Staatsgewalt nur mit einem Drittel der Mitglieder und die Vertreter der Arbeiter des Industriezweiges elurifalls nit einem Drittel vertreten seion, während die Vertreter der organisierten Abnohmer das letzte Drittel bilden sollten ). Auch Kautsky weiß wohl, daß zwischen den Interessen der Arbeiter und cler: Konsumenten nin gewisser (tegensatz besteht, da jene nach hohen Iölunen und kurzer Arbeitszeit, diesse nach niederen Preisen strében. Dieser Gegensatz wäre aber nuch seiner Ansicht durch den fortschritt zu einer hühoren l'roduktivitit der Arbeit $\mathrm{zn}$ überwinden, an der buide TGile gleichnn̈Big interassicri weien, und deshalb wird gefolgert: „Das gemeinsume Interesse der Arbeiter und Konsumenten wird den Stachel gur Erhichung der Produktivilät der Arbeiter setzen, den unter kapitulistischen Verhältnissen das Profitstreben des Unternehmers bildet."

Die Besitzer der verstaatlichten Betriebe will Kautsky zwar entschädigen, wat sehr gerecht klingt, jedoch praktisch dadurch stark eingeschränkt wird, da B bei der Entsohädigung außer dem Werte der Produktionsmittel Baulichkeiten, Maschinen, Rohstoffe usw. dio a uge nblic kliche Rentabilität nach Durchführung dor allgemeinen Soxialreformen berücksichtigt werden sollte. Wenn man nun bedenkt, wie schon seit der Revolution die Rentabilität, sofern sin tiberhaupt noch vorhauden ist, in den meigten Betrieben auBerondentlich stark gesunken ist, so wird man jenes Entgegenkounmen Kautsky s nicht als sehr weitgehend bexeichnen konnen. Be. merkenswerterweise wird kein Wort davon gesagt, was die Cnternehmer und Techniker an dom Aufbeu so vieler Werke geleistet haben. Gerade in der ohemischen Industrie wird man aber doch wohl nicht bohaupten können, daB dio Arbeiter in erster Linio dor doutechen chemischen Industrie ihre einstige Weitstellung verachafft haben. Man kann es daher auch durchaus verstehen, daB gegenwirtig so manche Unternehmer durch die Unvicherheit der Lage und vor allom auch durch dos ganze Sozialisierungegenohroi veranla Bt, in ihrer altgewohnten Energio etwa gelahmt worden oind, was nicherlich nicht als im Interesse der Industrio liegend bezeichnet worden Lain. Eino Durchfuhrung des Ka utskysohen Programma

") Wo aber bleiben dic technischen Betriebaleiter, ohno dio das ganzo Kollegium doch auf dis Daver niohts Wertrolles zu leisten vermag? wind jedenfallh einer groben Zahl von deutsohen Chemikern durcheno nicht als ein Akt der Gerechtigkeit orscheinen, sondern vielmehr ale ein verhängnievoller Vorgang, der letzten Fndes zum Ruin das Industrie führen muB. Wenn der Sozialismus auf dem Wege der Ka uts ky schen Richtlinion fortschreiten will, so wird er zweifellow gerado in rer shemiachen Industrie Deutschlands, auf die wir einstmals mit Recht so stolz gewesen sind, gerade dasjenige vernichten; was durch die Intelligene von Chenikern, Ingenieuren und Kaufleuten in langer, niühevoller Arbeit im Laufe von Jalirzehnten auf. gebaut worden ist. Vernichten lißst sich nun zwar eine Industrie verhältuismäßig leicht, aber der Wiederaufbau dürfte gerade unter den duroh den unglücklichen Ausgang des Krieges noch wesentlich verschärften schweren Konkurrenzverhälinissen auf dem Weltmarkte cin fast unlösbares Problem bilder. Deshalb kamu in der Tat dev so oft in leichtherzigel Weise erhobenen Forlerungen nach Sozialisiorung det deutschon Industrie, einschließlich der shemisoben Gewerbe, nur ein "Caveant consules" entgegengerufen werden.

Die deutschen Chemiker aber mögen sich in ihrer Gesamtheit etwas mehr als bisher rübren, damit sie nicht ebenfalls von der. Sturmflut fortgerissen werclen; deun wenn wan mit der Sozialisieruig des Bergbaucs, der der chemischen Industrie so vicle not: wendige Stoffe liefert, beginnen will, so wird man bald geilug auch von der Rohstoffgexiunnng zur Fertigfalurilation n̈bergehen wollen, deshalb: Discite moniti!

[A. 28.]

Chloroform- und acetonlösliche Celluloseacetate. Fin H. Css.

(Xingsog. 7./1. 1919.)

Die Chemie der Celluloscacetate ist vorwiegend in Patentiohriften niedergelegt, die vicle wissenwchaftliche Irrgen im Dunkel laween. Die Acetite sind in Zusammenseciung und Eigensehaften auBerordentlioh verschieden und lassen sich obensowenig unter den Gruppen der Tri- und Diacetato unterbringen, wie die Salpetensüureester unter 'Tri- und Dinitraten. Reine Triacetate mit dem aus der Formel bereohneten Essigsäuregehalte eshïll man mit Chlorzink als Katalysator; werden sie mit Schwefelsüure oder Sulfaten hergeatellt, so enthalten sie stets weniger Fssigsäure als Triscetat tind daftir Sulfoacetate beigemengt, was bisher mir und anderen Autoren entgangen war. In Löslichkeit und technischen Worte werden aie, auch bei gleichen Ansïtzen, durch kieine Anderungen der Temporatur und der Dauer des Acetylierens entscheidend beeinfluBt. Viele Acetate sind in den bcsten Lösungamitteln, alkoholhaltigem, Chloroforen und T'etrachlorïthan, nicht löslich, und die viel umstrittone, erst seit 1905-1910 bekannt gewordene Atetonlöslichkeit tritt nur bei bestimmten Umwandlungaprodukten primärer Acetato ein. Dir technisrhe Brauchbarkeit setat gute Löslichkeit und hohe Viscositäi der Jösungen voralis, so daß bei deren Verdunsten clustisobo: Films hinterbleiben, und die Herstellung solcher Acetate exfordert, trotz allar Putontheschreibungon, viel Firfahrung.

Line erschöpfende Behandlung des ganzen Ckebietes der Celluloseacetute, das in seinen Grundzigen abgreschlossen zu sein scheint, uberschreitet die Kräite eines einzelnen und wird noch Jahrzehnte? beanspruchen; ich habes aber in nehrjälurigen Studien die wichtigy sien Grappen durchgeuribeitet, um etwas Ordnung in ibre bunto Masse zu bringen, um die allgemeinen Kichtlinien ihrer Herstellungoweisen zu zithen und namentlich ihro allalytische Zusammen: setzung festzustellen. Die Angaben dor wichtigsten Patentsohrifted sind nachgeprift, und meine erste Arbuit über Cellulosercetate $\left.)^{1}\right)$ 1st wesentlich orweitert und in manohen Punkten berichtigt worden Nur diejenigen Acetate haben hier Beruicksichtigung gefunden, it denen das große Cellulosemolekul erhalten geblieben ist; dio be fortschreitender Acetolyse entstehenden Abbauprodulite, welol: schliestioh mebr als drei Acutyle auf eine $\mathrm{C}_{8}$-Gruppe dor Collulon. enthalten und richtiger als "Cellodextrinacetate" $\mathrm{zu}$ bezcichne" sind, welche mit zunchmender Molekulverkleinerung mehr und mol: in Aceton und in Alkohol löslich Werden und keine zusamma. bängenden Hilms liefern, sind anderswox) abgehandelt und im folge den ibergangen.

$$
\text { I. A } 11 \mathrm{~g} \text { e rne in es. }
$$

Zu allen Versuchen wurde eino und dieselbe, mit verdunnt Natronlauge gereinigte Baumwolle "für SchieBbaumwolle" $n$

1) Angew. Chemn. 19, 993 [1906].
H. O t , Liebigs Ann. 398, 314, 323 [1913]; Angew. Cbot 25. 1467 [1912]; Chem.-Ztg. sc, 1099 [1912]. 